PROCEEDINGS OF THE

AMERICAN MATHEMATICAL SOCIETY

Volume 51, Number 2, September 1975

\title{
AN INVARIANT IDEAL OF A GROUP RING OF A FINITE GROUP. II
}

\author{
J. S. HSIA AND ROGER D. PETERSON
}

ABSTRACT. The vanishing of the numerical invariant $\nu(G)$ of a finite group $G$ is linked to the existence of certain central annihilators of the generic right ideal $\Gamma_{R}(G)$ in the group ring $R G$. This leads to several affirmative answers of questions posed in [1]. Also, some explicit values of $\nu(G)$ are described for the class of finite nonsolvable groups having all their odd Sylow subgroups cyclic.

For an associative ring $R$ with identity and a finite group $G$, denote by $\Gamma_{R}(G)$ the right ideal of the group ring $R G$ generated by elements of the form $\sigma(H)=\Sigma_{h \in H} h$, where $H$ is a nontrivial subgroup of $G$. Since $g \sigma(H)=$ $\sigma\left(g g^{-1}\right) g$ and $r \sigma(H)=\sigma(H) r$, we see that $\Gamma_{R}(G)$ is also the left ideal of $R G$ generated by these $\sigma(H)^{\prime}$ 's. An easy coset argument shows that the generators $\sigma(H)$ for $\Gamma_{R}(G)$ may be restricted to the prime-order subgroups of $G$. The invariant ideal $\gamma_{R}(G)$ of $R$ with respect to. $G$ is then defined to be the (two-sided) ideal $R \cap \Gamma_{R}(G)$ [1]. When $R$ is che ring of rational integers, we put $\Gamma(G)=\Gamma_{Z}(G)$, and $\gamma(G)=\gamma_{Z}(G)=\nu(G) Z$ with $\nu(G) \geq 0$. This uniquely determines a nonnegative numerical invariant $\nu(G)$ for $G$; see also [3], [4]. In [1] three fundamental reduction theorems were proved, and using them, explicit theoretical as well as numerical results of the invariant for several large classes of groups were obtained. The present article continues the investigation for $\gamma_{R}(G)$. Recall a group is tight if it is a noncyclic group of order $p q$, where $p$ and $q$ are not necessarily distinct primes. In the solvable case, the numerical invariant is essentially determined by the numerical invariants of the tight subgroups (for instance, see [1, Theorem I.F.2]). This is generally not so in the nonsolvable case as $\operatorname{SL}(2, p)$ has no tight subgroups for $p$ a Fermat prime, and yet $0 \neq \nu(\operatorname{SL}(2, p))$ whenever $p \geq 17$, and also $p$ divides $\nu(\operatorname{SL}(2, p))$. See [1, Proposition I.G.4 and Appendix IIIc]. For nonsolvable groups, the precise determination of $\nu(\mathrm{SL}(2, p))$ for $p$ a Fer-

Received by the editors April 8, 1974.

AMS (MOS) subject classifications (1970). Primary 16A26, 20D99; Secondary 12A55, 20C05, 12B15.

Key words and phrases. Invariant ideal of a group ring, numerical invariant of a finite group, central annihilators, tight groups, condition (*), fixed-point-free representations. 
mat prime is of paramount importance. Scharlau showed in [4] that $\nu(\operatorname{SL}(2,5))=0$ because $\operatorname{SL}(2,5)$ admits a fixed-point-free representation over the rationals. With the aid of a computer, thanks to the efforts of David Ford, we answer here affirmatively a question raised in [1]; namely: $\Gamma(\operatorname{SL}(2,5))$ has a central annihilator $\alpha$ such that the coefficient coeff $\alpha\left(g_{0}\right)$ of $\alpha$ at $g_{0}$ is 1 , for some $g_{0} \in \operatorname{SL}(2,5)$. From this result follow several interesting consequences. In particular, we have:

(i). For all $R, \gamma_{R}(\mathrm{SL}(2,5))=0$;

(ii) $\nu\left(G_{1} \times G_{2}\right)=$ g.c.d. $\left(\nu\left(G_{1}\right), \nu\left(G_{2}\right)\right)$ if g.c.d. $\left(\left|G_{1}\right|,\left|G_{2}\right|\right)=1$.

Using theorems of Suzuki about the structures of groups all of whose odd Sylow subgroups are cyclic, we are able to determine in most cases the precise value for $\nu(G)$ where $G$ is a nonsolvable group whose Sylow subgroups all have vanishing numerical invariants. Again, the exceptional cases occur when $G$ contains a subgroup of the type $\operatorname{SL}(2, p), p$ a Fermat prime $\geq 17$. We conclude this introductory discussion with two conjectures:

(A) $\nu(\operatorname{SL}(2, p))=p$ for $p$ a Fermat prime $\geq 17$.

(B) If $G$ is a solvable group and $\nu(G)=1$, then $G$ contains tight subgroups with differing numerical invariants.

Central annihilators. As was observed by Scharlau [4, Satz 1], the vanishing of the numerical invariant for $G$ occurs precisely. when $G$ admits a fixed-point-free representation over the field of rationals. From the proof of this statement, it is clear that $\nu(G)=0$ if and only if $\Gamma_{\mathrm{Q}}(G)$ has a nonzero central annitilator $\alpha$ in $\mathbf{Q}$. On the other hand, the proof of $[1$, Theorem I. A] suggests that if $\nu(G)=0$ then $\Gamma_{R}(G)$ should more-or-less have a central annihilator in $R G$ with one of its coefficients equals to one. Indeed, we have

Theorem 1. Suppose $\nu(G)=0$. Then $G$ has a normal subgroup $G_{0}$ such that

(i) $G_{0}$ contains every prime-order subgroup of $G$, and

(ii) $\Gamma_{R}\left(G_{0}\right)$ has a central annibilator $\alpha$ in $R G_{0}$ with coeff ${ }_{a}\left(g_{0}\right)=1$ for some $g_{0} \in G_{0}$.

We first need two lemmas and a definition. A finite group $G$ is said to satisfy condition $(*)$ if $G$ has exactly one prime-order subgroup for each prime dividing the order $|G|$ of $G$.

Lemma 2. Suppose $\nu(G)=0$. Then $G$ has a normal subgroup $H$ such that $[G: H] \leq 2$, and $H \cong L \times M$ where $L$ satisfies condition $(*), M$ is either 1 , 
or $\operatorname{SL}(2,3)$, or $\operatorname{SL}(2,5), L$ and $M$ have relatively prime orders, and $H$ contains every prime-order subgroup of $G$.

Proof. The hypothesis $\nu(G)=0$ implies all the odd Sylow subgroups of $G$ are cyclic, and a Sylow 2-subgroup of $G$ is either cyclic or generalized quaternion (see [1, I.B.2] or [4, Satz 3]). Suzuki's the orem [5, Theorem C] implies then the existence of an $H$ with $[G: H] \leq 2$ with $H \cong L \times M$ where $L$ is a group all of whose Sylow subgroups are cyclic, and $M$ is either trivial or $\operatorname{SL}(2, p)$ for some prime $p$. As $M$ cannot have any tight subgroups it must be either trivial or else $p$ must be a Fermat prime (see [1, I.G.2]). And since $\nu(M)$ vanishes, $p$ can only be either 3 or $5 . L$ is solvable, so since $\nu(L)$ must also vanish, $L$ satisfies condition (*) from the proof of Theorem I.F.2 (Case i) of [1]. As $G$ has at most one involution, $H$ clearly contains every prime-order subgroup of $G$.

Lemma 3. If $K$ satisfies condition (*), then there is a subgroup $H$ which contains all the prime-order subgroups of $G$, and an element $\alpha$ in the center $§(R H)$ of $R H$, for any $R$, such that a annibilates $\Gamma_{R}(K)$ and $\operatorname{coeff}_{\alpha}(1)=1$.

Proof. If $H_{i}=\left\langle h_{i}\right\rangle, 1 \leq i \leq m$ are the distinct prime-order subgroups of $K$. Put $H=H_{1} \times \cdots \times H_{m}$, and $a=\left(1-b_{1}\right) \cdots\left(1-b_{m}\right)$. Then, $\alpha$ annihilates $\sigma\left(H_{i}\right)$ for every $i$, and so it annihilates $\Gamma_{R}(K)$. The coefficient of $\alpha$ at 1 is clearly 1 .

Proof of Theorem 1. Since the center of $\mathrm{ZG}$ is contained in the center of $R G$ and since $\Gamma_{R}(G)$ is the right ideal generated by elements of the form $\sigma(H)$, where $H$ runs through the prime-order subgroups of $G$, it suffices to consider the case when $R=\mathbf{Z}$. As $\nu(G)=0$, there exists a subgroup $H=$ $L \times M$ with properties described in Lemma 2 . Let $N$ be the subgroup of $L$ generated by its prime-order subgroups, so that $N$ is cyclic of square-free order, and consider the subgroup $G_{0}=N \times M$. Clearly, $G_{0}$ is normal in $G$ and contains every prime-order subgroup of $G$. By Lemma $3, \Gamma(N)$ has a central annihilator $\alpha$ in $Z N$ such that coeff $_{\alpha}(1)=1$. If $M=1$, we are done. If $M=\operatorname{SL}(2,3)$, then the computations given in $[1$, Appendix IIIa] show that $\Gamma(M)$ has a central annihilator $\beta$ in $Z M$ with : coeff ${ }_{\beta}\left(m_{0}\right)=1$ for some $m_{0} \in M$. With the aid of a computer (see [2]; also Appendix given below) the same result remains true for $M=\operatorname{SL}(2,5)$. Thus, $\alpha \beta$ is a central element of $Z G_{0}$, and it kills every prime-order subgroup $P$ of $G_{0}$ since every primeorder subgroup of $G_{0}$ lies either in $N$ or in $M$. Finally, coeff $\alpha \beta\left(m_{0}\right)=1$.

Corollary 4. If $\nu(G)=0$, then $\gamma_{R}(G)=0$ for all $R$. 
Proof. Let $G_{0}$ be a normal subgroup of $G$ such that $G_{0}$ contains every prime-order subgroup of $G$ and so $\Gamma_{R}\left(G_{0}\right)$ has a central annihilator $a$ with coeff $a\left(g_{0}\right)=1$ for some $g_{0} \in G_{0}$. By the first reduction theorem [1, Theorem I.D.2], $\gamma_{R}(G)=\gamma_{R}\left(G_{0}\right)$. On the other hand, if $r \in \gamma_{R}\left(G_{0}\right)$, then $r \cdot a=0$, so that $r=$ coeff $_{r a}\left(g_{0}\right)=0$. Thus, $\gamma_{R}(G)=\gamma_{R}\left(G_{0}\right)=0$.

Corollary 5. If $H$ and $K$ are groups of relatively prime orders, then $\gamma_{R}(H \times K)=\gamma_{R}(H)+\gamma_{R}(K)$. In particular, therefore, we have: $\nu(H \times K)=$ g.c.d. $(\nu(H), \nu(K))$.

Proof. If $\nu(H) \neq 0$, then every prime divisor of $\nu(H)$ divides $|H|$ [4, Satz 5]. In particular, if $\nu(H)$ and $\nu(K)$ both do not vanish, then $\nu(H) R+$ $\nu(K) R=R$, forcing $\gamma_{R}(H \times K)=R=\gamma_{R}(H)+\gamma_{R}(K)$. Hence, assume $\nu(H)=0$. Corollary 4 yields $\gamma_{R}(H)=0$. Also, $H$ has a normal subgroup $H_{0}$ which contains every prime-order subgroup of $H$ and such that $\Gamma_{R}\left(H_{0}\right)$ has a central annihilator $\alpha$ in $R H_{0}$ with coeff $a_{a}\left(h_{0}\right)=1$ for some $h_{0} \in H_{0}$. Since $H_{0} \times K$ contains every prime-order subgroup of $H \times K$, by the first reduction theorem [1, I.D.2], $\gamma_{R}(H \times K)=\gamma_{R}\left(H_{0} \times K\right)$. Hence, we may assume $H=H_{0}$. Suppose $r \in \gamma_{R}(H \times K)$. Write

$$
r=\sum_{h \in H} a_{h} \cdot h+\sum_{k \in K} b_{k} \cdot k
$$

where $a_{b} \in \Gamma_{R}(K)$ for all $b \in H$ and $b_{k} \in \Gamma_{R}(H)$ for all $k \in K$. Since $\alpha$ kills $b_{k}$ for all $k \in K$ and is central in $R(H \times K)$, we have

$$
\dot{r a}=\sum_{h \in H} a_{h} \cdot a \cdot h .
$$

Let $a=\Sigma_{b \in H} c_{b} \cdot b$ with $c_{b} \in R$ for all $b \in H$. Then,

$$
r a=\sum_{y \in H} r \cdot c_{y} \cdot y=\sum_{y \in H}\left(\sum_{b \in H} a_{b} \cdot c_{b^{-1} y}\right) \cdot y .
$$

But, $R(H \times K)$ is a free $R K$-module with basis $H$. Therefore,

$$
r \cdot c_{y}=\sum_{h \in H} a_{b} \cdot c_{h^{-1} y}
$$

belongs to $\Gamma_{R}(K)$ for all $y \in H$. As $c_{y}=1$ for some $y \in H$, this gives $r \epsilon$ $\gamma_{R}(K)$. Hence we have:

$$
\gamma_{R}(H \times K)=\gamma_{R}(K)=\gamma_{R}(H)+\gamma_{R}(K) .
$$

Corollary 6. Suppose $Q$ is a normal Sylow q-subgroup of $G$ for some prime $q$. If $\nu(G / Q)=0$, then we have: 
(i) $\nu(G)=0$ or $\nu(G)=q$.

(ii) $\nu(G)=$ g.c.d. $(\{\nu(T) \mid T$ is a tight subgroup of $G\})$.

(iii) $\gamma_{R}(G)=\nu(G) R$.

Proof. Let $H$ be a complement of $Q$ in $G$, and so $\nu(H)=0$. As before, we may suppose $H=H_{0}$ and there is a central annihilator $\alpha$ in $R H$ with coeff $a_{a}\left(b_{0}\right)=1$ for some $h \in H$. By the third reduction theorem [1, Theorem I.D.4], we have $\gamma_{R}(G)$ is contained in $R \cap\left(\Gamma_{R}(H)+q \cdot R H\right)$. If $r$ belongs to this intersection, write $r=a+q b$ with $a \in \Gamma_{R}(H)$ and $b \in R H$. Then, $r \cdot a=a \cdot a+q \cdot a \cdot b=q \cdot a \cdot b$. But then, $r=\operatorname{coeff}_{r \cdot a}\left(b_{0}\right)=\operatorname{coeff}_{q \cdot a_{0} b}\left(b_{0}\right)$ $\epsilon q R$. Hence, $\gamma_{R}(G) \subseteq q R$. In particular, if $G$ has a tight subgroup with numerical invariant $q$, then we are finished. So, assume that $G$ has no such tight subgroup. In particular, $Q$ is cyclic or $Q$ is generalized quaternion.

Case $1 . Q$ is cyclic.

Let $Q_{0}$ be the unique subgroup of $Q$ of order $q$. Then, $Q_{0} \triangleleft G$, and the centralizer $C_{G}\left(Q_{0}\right)$ must contain every prime-order subgroup of $G$ since otherwise $G$ would have a nonabelian tight subgroup having numerical invariant $q$. But, $C_{G}\left(Q_{0}\right)=Q \times C_{H}\left(Q_{0}\right)$. Using the first two reduction theorems [1, I.D.2, I.D.3], we deduce:

$$
\gamma_{R}(G)=\gamma_{R}\left(C_{G}\left(Q_{0}\right)\right)=\gamma_{R}\left(Q \times C_{H}\left(Q_{0}\right)\right)=\gamma_{R}\left(Q_{0} \times C_{H}\left(Q_{0}\right)\right)=\gamma_{R}\left(C_{H}\left(Q_{0}\right)\right) .
$$

Clearly, $\nu\left(C_{H}\left(Q_{0}\right)\right)=0$ since $\nu(H)=0$. Thus, $\gamma_{R}(G)=0$. Also, every tight subgroup of $G$ lies in $C_{H}\left(Q_{0}\right)$ and so in $H$, but $H$ has no tight subgroups.

Case 2. $Q$ is a generalized quaternion.

$G$ has a unique central involution. If $|Q|>8$, then $G / C_{G}(Q)$ is a 2-group, so that $C_{G}(Q)$ contains every prime-order subgroup of $G$. But, $C_{G}(Q)$ $=T \times C_{H}(Q)$, where $T$ is the central subgroup of $G$ of order two. This reverts back to Case 1 by the reduction theorems. Hence, we mav suppose that $|Q|=8$. As proved in [1], either $G=Q \times H$ with $H$ satisfying condition $(*)$ and also being of odd order or else we have $G=\operatorname{SL}(2,3) \times H$ with $H$ satisfying condition $(*)$ and $6 . \nmid|H|$. In either case, $G$ is solvable and has no tight subgroups so that $\nu(G)=0$. Thus we are done by $[1$, Theorem I.F.2].

Remark. Corollary 4 follows also from Corollary 6(iii) and the second reduction theorem. Indeed, if $\nu(G)=0$, choose a prime $q$ not dividing $|G|$. Consider $\tilde{G}=G \times Q$ where $Q \cong \mathrm{Z} / q \mathrm{Z}$. So, we have $\gamma_{R}(G)=\gamma_{R}(\tilde{G})=\nu(\tilde{G}) R=$ $\nu(G) R=0$.

Nonsolvable groups having cyclic odd Sylow subgroups. In the setting here $\nu(S)$ vanishes for all odd Sylow subgroups $S$ of $G$. We shall consider 
two cases: (a) a Sylow 2-subgroup is generalized quaternion, and (b) a Sylow 2-subgroup is dihedral. In both these cases, Suzuki's structural theorems apply; see [5].

Case (a). $G$ has subgroup $G_{0}$ of index at most two and $G_{0}$ is isomorphic to $H \times \mathrm{SL}(2, p)$, where $H$ is a Zassenhaus group (i.e. every Sylow subgroup of $H$ is cyclic) and $p$ a prime $\geq 5$, since $G$ is nonsolvable. In particular, we observe that the orders of $H$ and $\operatorname{SL}(2, p)$ are relatively prime. Also,as $G$ has a unique involution, $G_{0}$ contains every prime-order subgroup of $G$ so that $\gamma_{R}(G)=\gamma_{R}\left(G_{0}\right)$. Since $H$ is solvable and all of its Sylow subgroups have vanishing numerical invariants, Theorem I.F.2 [1] can be applied to determine $\gamma_{R}(H)$. In particular, $\nu(H)$ is either 0,1 , or a prime dividing $|H|$. If $p$ is not a Fermat prime, then $\nu(\operatorname{SL}(2, p))=p$ (see [1, Corollary I.G.5]). Therefore, we deduce:

$$
\nu(G)=\text { g.c.d. }(\nu(H), p)=\text { g.c.d. }(\{\nu(T) \mid T \text { is a tight subgroup of } G\}) .
$$

If $p$ is a Fermat prime and if $\nu(H)=0$ (equivalently, $H$ has no tight subgroups), then $\nu(G)=\nu(\operatorname{SL}(2, p))$ by the reduction theorems. Of course, when $\nu(H)=1, \nu(G)=1$ as well. On the other hand, if $\nu(H)=q$, then $q+|\operatorname{SL}(2, p)|$. As $\nu(\mathrm{SL}(2, p)) \neq 0$ for $p>5$, we conclude: $\nu(G)=1$ for $p>5$, and $\nu(G)=q$ when $p=5$. Thus, if the precise value of $\nu(\operatorname{SL}(2, p))$ can be achieved for Fermat primes exceeding 5 , the precise value for $\nu(G)$ can also be completely determined.

Case (b). Again, Suzuki's Theorem B [5] gives us the structure for $G$; namely, $G$ contains a normal subgroup $G_{0}$ of index at most two, and $G_{0} \cong$ $H \times \operatorname{LF}(2, p)$ where $H$ is a Zassenhaus group and $\operatorname{LF}(2, p)$ is the linear fractional group with $p \geq 5$. It is well known that $\operatorname{LF}(2, p) \cong \operatorname{PSL}(2, p)$ are simple groups, and so by $\left[1\right.$, Corollary I.C.3] we have $\gamma_{R}(\operatorname{LF}(2, p))=R$ for all $R$. Thus, $\gamma_{R}(G)=R$.

Remark. It is well known that a finite group all of whose abelian subgroups are cyclic is precisely a group which has each of its Sylow subgroups being either cyclic or generalized quaternion; in other words, precisely when all the Sylow subgroups have vanishing numerical invariants. A theorem of Artin-Tate then says such a group is exactly one that has periodic cohomology groups. Such groups are also of interest in topology where the groups operate on spheres fixed-point-freely.

If we wish to include the Klein 4-group as a dihedral group, then the discussion given in Case (b) remains valid. For, Suzuki's Theorem A [5] implies $G \cong H \times \operatorname{LF}(2, p)$ once again (for $p \geq 5)$ and the simplicity of $\operatorname{LF}(2, p)$ yields $\gamma_{R}(G)=R$. 
Appendix. We present here the summary results of a computer-assisted computation for the central annihilators of $\Gamma(\operatorname{SL}(2,5))$. Denote by $\langle a, b, c, d\rangle$ the group element $\left(\begin{array}{ll}a & b \\ c & d\end{array}\right)$, and by $\operatorname{ccl}(y)$ the group ring element formed by summing the members in the conjugacy class of $y$.

There are nine conjugacy classes, and a $\mathrm{Z}$-module basis for the center $\hat{Z}(\mathbf{Z} G)$ is given by:

$$
\begin{aligned}
& \{R=1=\langle 1,0,0,1\rangle, S=\langle-1,0,0,-1\rangle, T=\operatorname{ccl}(\langle 3,2,2,0\rangle), \\
& U=\operatorname{ccl}(\langle 2,2,2,0\rangle), V=\operatorname{ccl}(\langle 3,4,1,0\rangle) ; W=\operatorname{ccl}(\langle-1,-1,1,0\rangle), \\
& X=\operatorname{ccl}(\langle 0,-1,1,0\rangle), Y=\operatorname{ccl}(\langle 1,-1,1,0\rangle), \text { and } Z=\operatorname{ccl}(\langle 2,-1,1,0\rangle)\}
\end{aligned}
$$

If $\mathscr{U}$ denotes the left annihilator ideal in $\mathrm{Z} G$ for $\Gamma(G)$, then a $\mathrm{Z}$-module basis for $Z(Z G) \cap \mathscr{Q}$ is:

$$
\{A=2 R-2 S+T-U-W+Y, \quad B=T-U-V+Z=(S-R)(U-Z)\}
$$

\section{REFERENCES}

1. J.S. Hsia and R. D. Peterson, An invariant ideal of a group ring of a finite group, and applications, J. Algebra 32 (1974), 576-599.

2. David Ford, J. S. Hsia and R. D. Peterson, Some computations of the numerical invariant of a finite group (in preparation).

3. M. Knebusch and W. Scharlau, Über das Verhalten der Witt-Gruppe bei galoischen Körpererweiterungen, Math. Ann. 193 (1971), 189-196. MR 45 \#1955.

4. W. Scharlau, Eine Invariante endlicher Gruppen, Math. Z. 130 (1973). 291-296.

5. M. Suzuki, On finite groups with cyclic Sylow subgroups for all odd primes, Amer. J. Math. 77 (1955), 657-691. MR 17, 580.

DEPARTMENT OF MATHEMATICS, OHIO STATE UNIVERSITY, COLUMBUS, OHIO 43210 (Current address of J.S. Hsia)

Current address (R. D. Peterson): Department of Mathematics, University of Wisconsin, Milwaukee, Wisconsin 53201 